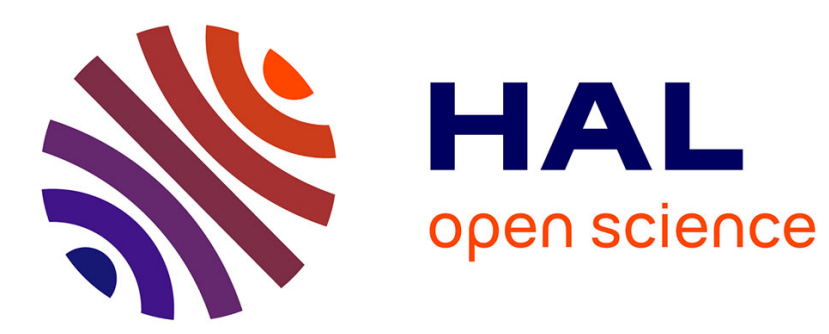

\title{
Modeling and optimal force control of a nonlinear electrostatic microgripper.
}

\author{
Mokrane Boudaoud, Yassine Haddab, Yann Le Gorrec
}

\section{To cite this version:}

Mokrane Boudaoud, Yassine Haddab, Yann Le Gorrec. Modeling and optimal force control of a nonlinear electrostatic microgripper.. IEEE/ASME Transactions on Mechatronics, 2013, 18, pp.11301139. hal-00874563

\section{HAL Id: hal-00874563 https://hal.science/hal-00874563}

Submitted on 18 Oct 2013

HAL is a multi-disciplinary open access archive for the deposit and dissemination of scientific research documents, whether they are published or not. The documents may come from teaching and research institutions in France or abroad, or from public or private research centers.
L'archive ouverte pluridisciplinaire HAL, est destinée au dépôt et à la diffusion de documents scientifiques de niveau recherche, publiés ou non, émanant des établissements d'enseignement et de recherche français ou étrangers, des laboratoires publics ou privés. 


\title{
Modeling and optimal force control of a nonlinear electrostatic microgripper
}

\author{
Mokrane Boudaoud, Yassine Haddab, IEEE Member, and Yann Le Gorrec
}

\begin{abstract}
Microgrippers with integrated force sensors are very efficient tools for dexterous manipulation of objects in the microworld (size less than $100 \mu \mathrm{m}$ ). In this paper, we first propose a modeling approach of a nonlinear electrostatic microgripper with integrated force sensor while handling calibrated micro-glass balls of $80 \mu \mathrm{m}$ diameter. Limit of the linear operating range of the microgripper is investigated and a nonlinear model is proposed and validated experimentally for large displacements. We then propose the design of an optimal force feedback controller to ensure reliable handling operations with appropriate gripping forces. To overcome the limitation caused by the low signal to noise ratio provided by the sensor, a Kalman filter is used to estimate the states of the process from noise measurements. The control law is implemented and validated using real time experiments for $10 \mu N$ gripping force reference with a noise level (peak-to-peak magnitude of the noise) reaching $8 \mu \mathrm{N}$ in the worst case. The effectiveness of the optimal filter is proven by comparison with external interferometric measurements.
\end{abstract}

Index Terms-Electrostatic microgripper, Nonlinear modeling, Noise analysis, Kalman filtering, LQG force control, Micro-glass balls manipulation.

\section{INTRODUCTION}

Designing and controlling systems able to perform dexterous manipulation tasks on small components (i.e. between $1 \mu \mathrm{m}$ and $1 \mathrm{~mm}$ ) is still a great scientific and technological challenge due to the influence of the surrounding environment and the difficulty of performing experiments in the microworld. The dexterous manipulation of micro-objects is often needed in microassembly [1], minimally invasive surgery, genetics and cell mechanical characterization [2] [3]. Such applications require the use of miniaturized grippers endowed with actuators compatible with the microworld specifications (small dimensions and large displacements) [4] [5] and force sensors for the measurement of gripping forces [6] [7].

To control the gripping force, a reliable modeling of the microgripper and the manipulated object is required. Knowledge based models are in this case relevant and give a physical significance to some phenomena such as nonlinear behaviors. Such models are nevertheless quite difficult to validate experimentally due to many problems commonly encountered in microrobotics such as: complex architectures and parameters uncertainties [8] due to the microfabrication process. To avoid such constraints, empirical models (black box models) are

The authors are with the FEMTO-ST Institute, Mixed Unit of Research (UMR) National Center for Scientific Research (CNRS) 6174, University of Franche-Comté (UFC)/National School of Mechanics and Microtechnology Besançon (ENSMM)/University of Technology of Belfort- Montbeliard (UTBM), Besançon 25000, France (mokrane.boudaoud, yassine.haddab, legorrec)@femto-st.fr. often used [9] with no information about internal properties of the modeled system. Advantages of empirical models is that they are simple and they can be easily obtained. However, with such models, the physical meaning of the system is lost.

In addition to the modeling part, the gripping force control requires dealing with the low signal to noise ratio of measurements commonly found at these scales. Indeed, it is very difficult to achieve controlled gripping forces when the magnitude of the desired force is close to the noise level (peakto-peak magnitude of the noise). Some successful attempts of gripping force control using microgrippers are obtained at the MilliNewton [10] [11], the MicroNewton [12] and the NanoNewton [13] force levels. In most cases, the modeling of the microgrippers is performed using linear empirical approaches and the limitation due to the weak signal to noise ratio is bypassed by working well above the noise level.

In this paper, we focus our attention on the modeling and the control of an electrostatic microgripper (FT-G100) (Fig1) designed by FemtoTools GmbH company. This microgripper integrates an electrostatic comb drive actuator characterized by a nonlinear behavior when performing large displacements [14] and a capacitive force sensor ( $50 \mathrm{nN}$ resolution) providing a noise signal with a peak-to-peak magnitude reaching $2.35 \mu \mathrm{N}$ in static mode. The motivation of the work reported in this paper is then twofold: 1) from a modeling point of view, we propose a knowledge based model able to capture the nonlinear behavior of a complex suspension system leading to the description of the dynamic behavior of the microgripper in a wide range of operating conditions. The accuracy of the nonlinear model is discussed based on experimentations. 2) from a control point of view, we propose a strategy able to deal with real time constraints and significant noises at such scale. Noises are characterized and the effectiveness of a Kalman filter for the estimation of the gripping force despite significant measurement noises is proven. The paper shows that a standard control strategy with a Kalman filter can be efficiently applied for accurate manipulation at the micro-scale.

The paper is organized as follow: first, the global architecture and the basic features of the FT-G100 microgripper are presented. After that, a modeling approach of the actuation system is proposed. Limit of the linear displacement range is investigated and a nonlinear model is derived and validated experimentally. Thirdly, in order to achieve a gripping force control, a coupled model of the microgripper is proposed when the end effectors are closed around a glass ball of $80 \mu \mathrm{m}$ diameter. In the fifth part, a discrete Kalman filter is computed using the coupled model and is tuned taking into account measurement and process noises which are beforehand characterized. Gripping force control is achieved in the sixth part 
TABLE I

DIMENSIONS OF THE ACTUATION AND SENSING SYTEMS

\begin{tabular}{l||lll}
\hline & actuated and sensing arms & clamped clamped suspension mechanism (actuator) & clamped clamped suspension mechanism (sensor) \\
\hline Length & $L=5150 \mu \mathrm{m}$ & $L_{s}=915 \mu \mathrm{m}$ (half length) & $L_{s b}=435 \mu \mathrm{m}$ (half length) \\
\hline Width & $l=150 \mu \mathrm{m}$ & $l_{s}=50 \mu \mathrm{m}$ & $l_{s b}=50 \mu \mathrm{m}$ \\
\hline Thickness & $e=50 \mu \mathrm{m}$ & $e_{s}=8 \mu \mathrm{m}$ & $e_{s b}=8 \mu \mathrm{m}$
\end{tabular}

using LQG (Linear Quadratic Gaussian) synthesis in which the gripping force is estimated in dynamic and static modes despite the noise. Results show successful force control with $10 \mu N$ gripping force reference with a noise level reaching $8 \mu N$ in the worst case. The effectiveness of the Kalman filter is proved using a high performance laser interferometer sensor.

\section{PResentation AND WORKING PRINCIPLE OF THE FT-G100 MICROGRIPPER}

The FT-G100 microgripper is designed to handle objects ranging from $1 \mu \mathrm{m}$ to $100 \mu \mathrm{m}$. To pick up an object, an actuated arm is pushed toward closure thanks to a comb drive actuator. The base of the actuated arm is fixed to a flexure joint (Fig1) and a suspension mechanism including two pairs of clampedclamped beams holds the movable part of the actuator. While the gripper arms are closed around an object, the deflection of the sensing arm is detected by a capacitive sensor. The sensor consists of a transverse comb-drive with a differential capacity proportional to the displacement of the movable fingers. This displacement (due to the gripping force) is translated into analog voltage $V_{\text {out }}$ throughout a MS3110 readout chip (Irvine Sensors) [15]. Two pairs of clamped-clamped beams and a flexure joint are also attached to the sensor and the base of the sensing arm respectively. Dimensions of the actuated and sensing arms and those of each clamped-clamped suspension are described in TABLE I. Benefits and shortages of the FTG100 microgripper can be found in [16].

\section{ACTUATION SYSTEM MODELING}

The actuation system (comb drive actuator + actuated arm) is considered to be made up of a linear and a nonlinear part. The nonlinear behavior of the clamped clamped suspensions is derived analytically and validated experimentally. Then a dynamic model is computed in a large working range.

\section{A. Derivation of the nonlinear behavior of the actuation system}

When applying an actuation voltage $V_{i n}$ to the comb drive actuator, an electrostatic force $F_{\text {elec }}$ is generated and is applied at the middle of the clamped-clamped suspensions (Fig2a). The general formulation of such a force is [17]:

$$
F_{\text {elec }}=\frac{N_{a} \cdot \varepsilon \cdot h_{z}}{2 . g} \cdot V_{i n}^{2}
$$

Where $N_{a}=1300$ is the total number of fingers (movable and fixed) in the actuator, $\varepsilon=8.85 \mathrm{pF} / \mathrm{m}$ is the permittivity of the dielectric material (air), $h_{z}=50 \mu m$ is the thickness of comb fingers, and $g=6 \mu \mathrm{m}$ is the gap spacing between two fingers.

Unlike conventional linear approaches, in nonlinear theory, when an external force is applied at the middle of a clampedclamped beam, axial forces $\vec{N}$ are developed in clamped parts

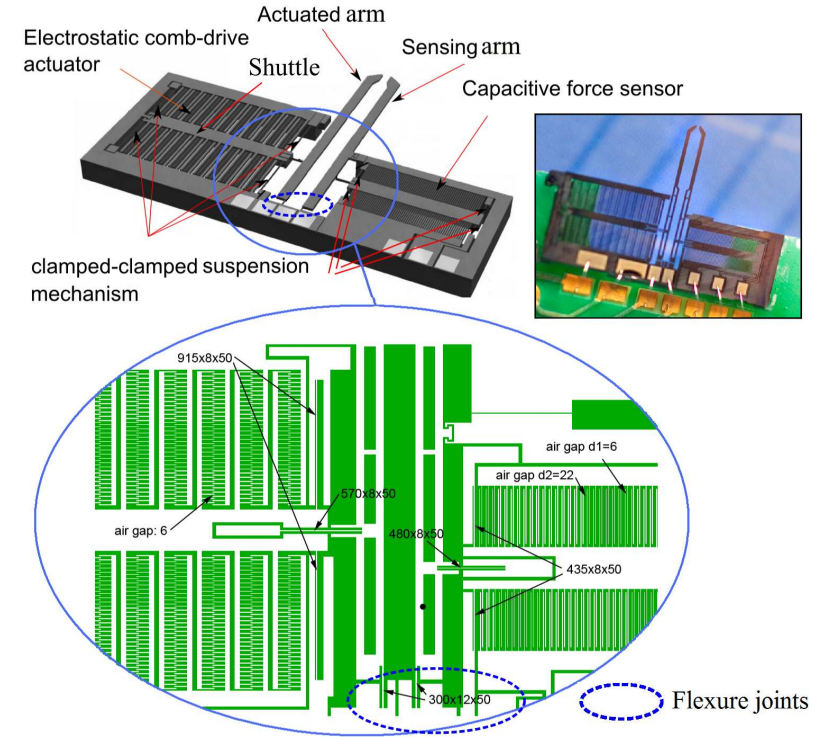

Fig. 1. Structure of the FT-G100 microgripper (FemtoTools GmbH). In the close-up view (part encircled by the ellipse with a solid line), the green color and the white color show the hollow areas and the body parts of the microgripper respectively.

of suspensions (Fig2a). Axial forces become significant in the case of large deflections of the beam. In the microgripper, let us consider the two clamped clamped suspensions of thickness $e_{s}$ on which the displacement $y_{a}\left(x_{e a}\right)$ in the direction of $y$ axis is governed by the nonlinear expression [18]:

$$
\begin{gathered}
y_{a}\left(x_{e a}\right)=e_{s} \sqrt{\frac{2}{3}} \cdot(u-\tanh (u)) \cdot Q(u) \\
Q(u)=\left(\frac{3}{2}-\frac{1}{2} \cdot(\tanh (u))^{2}-\frac{3}{2} \cdot \frac{\tanh (u)}{u}\right)^{-1 / 2} \\
u=\sqrt{\frac{N}{E \cdot I_{s}}} \cdot\left(\frac{L_{s}}{2}\right)
\end{gathered}
$$

Where $E=190 G P a$ is the Young's modulus of silicon type material and $I_{s}=2.13 \times 10^{-21} \mathrm{~m}^{4}$ is the area moment of inertia of the suspension's cross section.

The lateral stiffness (in the direction of $y$ axis) of the pseudo rigid body (i.e. actuated arm + flexure joint) is calculated in APPENDIX A. Due to the relatively low value of this stiffness $(0.8 \mathrm{~N} / \mathrm{m})$, it is assumed that the flexure joint behaves as a hinge joint. Thus, the actuated arm tip displacement $y_{a}(L)$ in the direction of $y$ axis can be deduced from $y_{a}\left(x_{e a}\right)$ such as:

$$
y_{a}(L)=\frac{L}{x_{e a}} \cdot y_{a}\left(x_{e a}\right)
$$

With $x_{e a}=1100 \mu m$ (see Fig2a)

The relation between an applied force at the middle of a clamped-clamped beam and the axial force $N$ can be derived from equilibrium of moments [19]. In the case of the 
electrostatic force $F_{\text {elec }}$ applied on the suspension mechanism, we have used the following formulation:

$$
F_{\text {elec }}=\frac{16 \cdot E \cdot I_{s}}{L_{s}^{3}} \cdot e_{s} \sqrt{\frac{2}{3}} \cdot u^{3} \cdot Q(u)
$$

Using equations (2) and (6), the nonlinear stiffness governing the relationship between the electrostatic force and the deflection $y_{a}\left(x_{e a}\right)$ can be derived as:

$$
K_{n l}=\frac{16 \cdot E \cdot I_{s}}{L_{s}^{3}} \cdot \frac{u^{3}}{u-\tanh (u)}
$$

Then, the stiffness $K_{n l}$ depends on the variable $u$. The latter also depends on the supply voltage $V_{\text {in }}$ through equations (6) and (1). Let us recall that the aim of this modeling is to provide
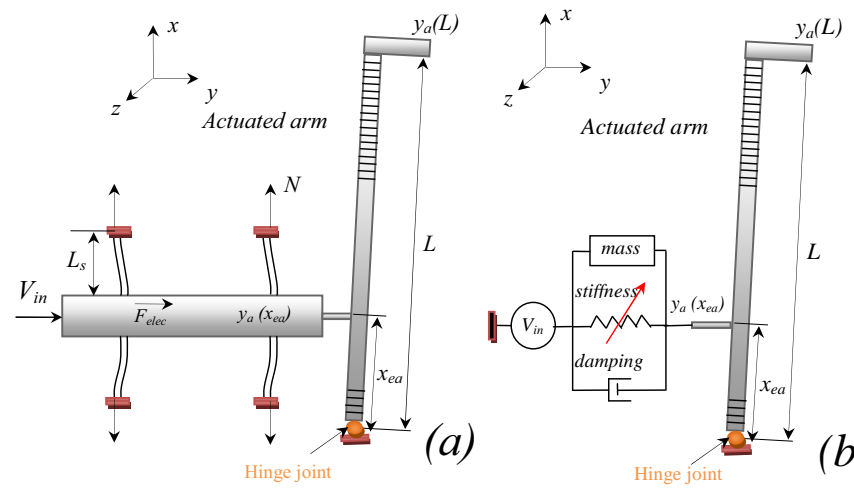

(b)

Fig. 2. Simplified scheme of the FT-G100 actuation system (a) and equivalent scheme of suspension mechanism (b).

models for control in which the input is $V_{i n}$ and the output relates to $y_{a}(L)$. For this purpose, it is necessary to express $K_{n l}$ in terms of $V_{i n}$. Then, in order to obtain the function $u=f\left(V_{i n}\right)$, the following equation has to be solved:

$$
V_{i n}=\left(\frac{2 \cdot g}{N_{a} \cdot \varepsilon \cdot h_{z}} \cdot \frac{16 \cdot E \cdot I_{s}}{L_{s}^{3}} \cdot e_{s} \cdot \sqrt{\frac{2}{3}} \cdot u^{3} \cdot Q(u)\right)^{1 / 2}
$$

Instead of analytically expressing $u=f\left(V_{i n}\right)$, we numerically solve equation (8). Starting from a data vector containing values of $V_{i n}$ from 0 to $200 \mathrm{~V}$ with $0.1 \mathrm{~V}$ steps, equation (8) has been solved using the Matlab fsolve function, and a vector of solutions containing values of $u$ has been obtained. Thus, based on Lagrange polynomials, an interpolation of the curve describing $u$ in terms of $V_{i n}$ led to:

$$
\begin{aligned}
u & =-\left(11.8324 \times 10^{-14} \cdot V_{i n}^{6}\right)+\left(5.7677 \times 10^{-11} \cdot V_{i n}^{5}\right) \\
& -\left(6.0704 \times 10^{-9} \cdot V_{i n}^{4}\right)-\left(11.4396 \times 10^{-7} \cdot V_{i n}^{3}\right) \\
& +\left(0.0002722 . V_{i n}^{2}\right)-\left(0.0018 \cdot V_{i n}\right)+0.0071
\end{aligned}
$$

Substituting equation (9) in (7), the nonlinear stiffness-supply voltage relation is obtained and shown in (Fig3).

Moreover, using equation (9) in (2) and (5), the tip displacement-supply voltage relation is derived and shown in (Fig4). On the same figures are presented the stiffness of the suspensions and the actuated arm tip displacement according to the linear theory (i.e. the stiffness does not depend on the supply voltage). The linear stiffness of the suspensions is [20]: $K_{l}=\frac{48 . E \cdot I_{s}}{L_{s}^{3}}$. This stiffness can be deduced from (7) when $u \rightarrow 0$.
Experimental measurements of the actuated arm tip displacements have been performed using a high resolution ( 0.01 $\mathrm{nm}$ ) laser interferometer (SP-120 SIOS Metechnik GmbH), when actuation voltages varying from 0 to $200 \mathrm{~V}$ (40 measurements) are applied to the actuator. Results are presented in (Fig4) illustrating a good accordance between experimental and theoretical approaches when using the nonlinear formulation. However, the linear stiffness theory is valid only for actuation voltages lower than $30 \mathrm{~V}$ which corresponds to $y_{a}(L)=7.86 \mu \mathrm{m}$.

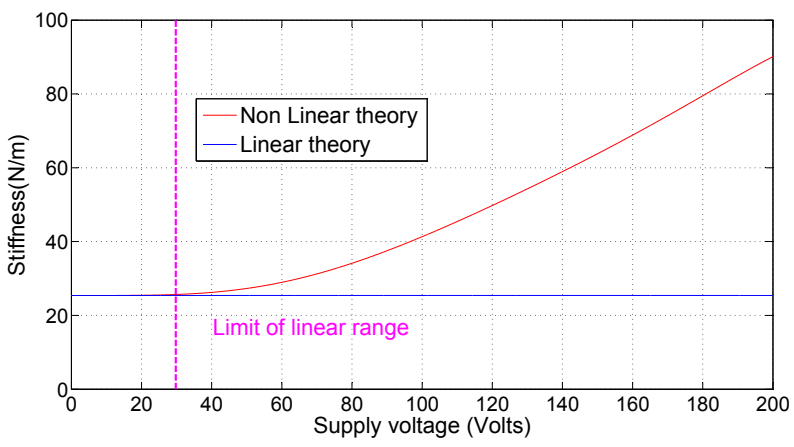

Fig. 3. Linear and nonlinear stiffness/supply voltage characteristic according to the suspension mechanism. The dashed line corresponds to $K_{n l}=1 \% . K_{l}$

The mean error of the nonlinear static characteristic in the whole operating range $\left(0<V_{i n}<200 \mathrm{~V}\right)$ is equal to $10.96 \%$. A variation of $1 \%$ of the expression $\frac{N_{a} \cdot \varepsilon \cdot h_{z}}{2 \cdot g}$ leads to an increase of $1.11 \%$ of the mean error of the static characteristic. The latter is increased by $1.13 \%$ when the variation of the nonlinear stiffness $K_{n l}$ at each operating point is about $1 \%$.

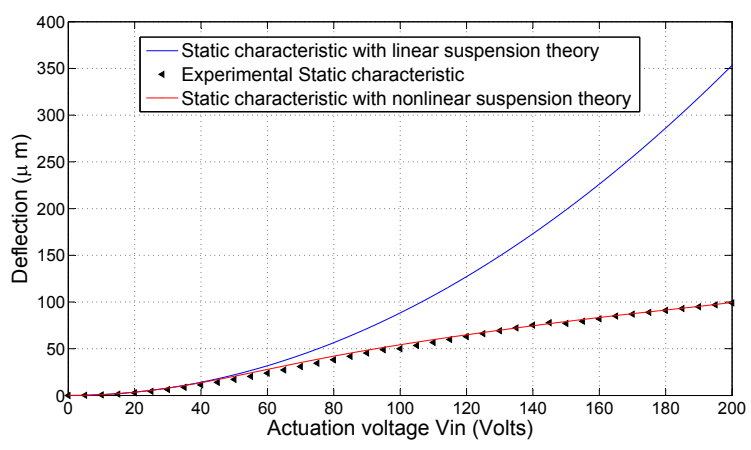

Fig. 4. Linear and nonlinear tip displacement/supply voltage characteristic of the actuation system

According to (Fig4), the nonlinear formulation is needed to describe the position of the tip of the actuated in a large operating range during its approach toward and object. Indeed, if the size of the object is ranging from $92 \mu \mathrm{m}$ to $100 \mu \mathrm{m}$, the stiffness of the actuation system can be described using the linear theory. However, for an object with a size lower than $92 \mu m$, the nonlinear theory must be used. Here, we refer just to the stiffness of the actuation system before its contact with an object. The effect of the gripping force on the overall stiffness of the actuation system is taken into account in section IV.C.

\section{B. Dynamic modeling}

Let us consider the displacement $y_{a}(x, t)$ in the direction of $y$ axis, of a point of coordinate $x(0<x<L)$ on the actuated 
arm. The partial differential equation describing the dynamic behavior $y_{a}(x, t)$ is given as:

E.I. $\frac{\partial^{4} y_{a}(x, t)}{\partial x^{4}}+\sigma \cdot \frac{\partial y_{a}(x, t)}{\partial t}+$ o.l.e. $\frac{\partial^{2} y_{a}(x, t)}{\partial t^{2}}=F(x, t) . \delta\left(x-x_{e a}\right)$

$I=1.4 \times 10^{-17} \mathrm{~m}^{4}$ is the area moment of inertia of the actuated arm, $\sigma$ is the damping factor, $\rho=2330 \mathrm{Kg} / \mathrm{m}^{3}$ is the mass density, $F(x, t)$ is a load per unit length applied on the actuated and $\delta\left(x-x_{e a}\right)$ is the Dirac delta function.

Equation (10) can be solved considering the following boundary conditions:

$$
\left\{\begin{array} { l } 
{ y _ { a } ( x _ { 0 } ) = 0 } \\
{ \text { E.I. } \frac { \partial ^ { 2 } y _ { a } ( x _ { 0 } ) } { \partial x ^ { 2 } } = 0 }
\end{array} \quad \left\{\begin{array}{l}
\text { E.I. } \frac{\partial^{2} y_{a}\left(x_{n_{b}}\right)}{\partial x^{2}}=0 \\
\text { E.I. } \frac{\partial^{3} y_{a}\left(x_{n_{b}}\right)}{\partial x^{3}}=0
\end{array}\right.\right.
$$

Assuming the actuated arm as a rigid body, equation (10) can be simplified to a lumped parameter model (mass-springdamper) with a nonlinear stiffness (see Fig2b):

$$
M_{a} \cdot \frac{\mathrm{d}^{2} y_{a}(L, t)}{\mathrm{d} t^{2}}+\sigma_{a} \cdot \frac{\mathrm{d} y_{a}(L, t)}{\mathrm{d} t}+\left(\frac{x_{e a}}{L}\right) \cdot K_{n l} \cdot y_{a}(L, t)=F_{\text {elec }}
$$

Where : $M_{a}$ is the mass of the actuation system (shuttle (Fig1) + suspensions + actuated arm). and $\sigma_{a}$ is the generalized damping of the system.

In equations (11), we have assumed that the slander beam with dimension $(570 \mu m \times 8 \mu m \times 50 \mu m)$ (see Fig1) is rigid and it is not subject to buckling. The reason is that the critical axial load [21] beyond which the beam begins to buckle is evaluated at $50 \mathrm{mN}$. This critical load cannot be reached in the studied microgripper. Indeed, as it will be shown in section IV.A, the maximum gripping force that can be applied to an object by the microgripper is about $120 \mu N$. In this case the slender beam is subject to an axial load equal to $562 \mu N<<50 \mathrm{mN}$.

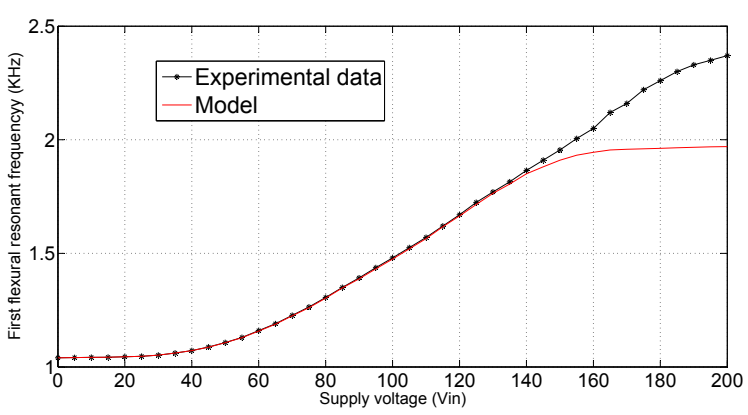

Fig. 5. Increase of the first flexural resonant frequency of the actuation system with increasing the supply voltage

To validate the model with the previous given assumptions, the mass $M_{a}$ and the damping $\sigma_{a}$ are identified. Therefore, a $5 \mathrm{~V}$ step excitation is applied to the actuation system and the resulting step response is used for the identification. In the operating range $0<\operatorname{Vin}<130 \mathrm{~V}$, the maximum error between the experimental resonance frequency of the actuation system and the one obtained from the model is less then $12.27 \%$. This error is more important when exceeding $130 \mathrm{~V}$. For this reason, the nonlinear model is validated only for $0<$ Vin $<130 \mathrm{~V}$ and the difference between the experimental and theoretical resonance frequency is compensated by applying a correction factor to the mass $M_{a}$. Results are presented in Fig5.

\section{COUPLED MOdEL OF THE MICROGRIPPER}

For force control, a coupled model of the gripper is used when the gripper arms are in contact with the microobject (Fig6). The coupled model includes the dynamics of the actuation and sensing systems through the stiffness of the manipulated object. Therefore, the nonlinear model of the actuation system is linearized around an operating point $\left(V_{i n_{0}}, y_{a_{0}}\right)$, the dynamic model of the sensing system is derived from experiments and the coupled model is computed.

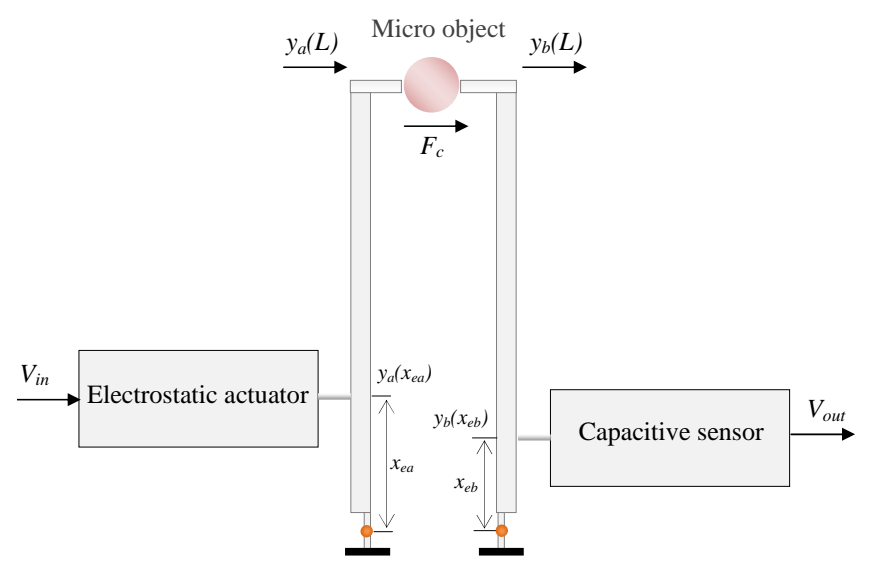

Fig. 6. Gripping system to be modeled

\section{A. Linearization of the actuation system's model}

A linear model of the actuation system is derived around an operating point $V_{i n_{0}}=60 \mathrm{~V}$ using a Jacobian linearization. The linear state space equation of the actuation system is:

$$
\begin{aligned}
& \left\{\begin{array}{l}
\dot{\tilde{X}}_{a}=\left[\begin{array}{cc}
0 & 1 \\
-\frac{K_{l a}}{M_{a}} & -\frac{\sigma_{a}}{M_{a}}
\end{array}\right] \cdot \tilde{X}_{a}+\left[\begin{array}{l}
0 \\
\left(\frac{L}{x_{e a}}\right) \frac{N_{a} \cdot \varepsilon \cdot h_{z}}{g \cdot M_{a}} \cdot V i n_{0}
\end{array}\right] \cdot \tilde{V}_{i n} \\
\tilde{y}_{a}(L, t)=\left[\begin{array}{ll}
1 & 0
\end{array}\right] \cdot \tilde{X}_{a}
\end{array}\right. \\
& \tilde{X}_{a}=\left[\begin{array}{ll}
\tilde{y}_{a}(L, t) & \left.\dot{\tilde{y}}_{a}(L, t)\right], \quad K_{l a}=K_{n l}\left(y_{a_{0}}\right)+\frac{d K_{n l}}{d y_{a}} \cdot y_{a_{0}}
\end{array}\right.
\end{aligned}
$$

$\tilde{X}_{a}, \tilde{y}_{a}(L, t)$, and $\tilde{V}_{i n}$ are the variation of the state vector, the free end deflection and the supply voltage respectively around the operating point. The linearized stiffness $K_{l a}$ is selected such as $K_{n l}\left(y_{0}\right)=28.95 \mathrm{~N} / \mathrm{m}$ and $\frac{d K_{n l}}{d y_{a}} y_{a_{0}} \approx 8.6 \mathrm{~N} / \mathrm{m}$

\section{B. Sensing system modeling and identification}

The static gripping force/arm tip displacements characteristic of the sensing system is extracted experimentally aiming at determining if a nonlinear behavior occurs for gripping forces ranging from few $\mu N$ up to $120 \mu N$. A rigid beam is used to perform displacements at the tip of the sensing arm (Fig7).

Tip displacements are recorded using the laser interferometer previously cited. Therefore, we have used the output voltage of the force sensor to evaluate each force corresponding to known displacements of the arm tip. As a result (Fig8), a linear characteristic can be observed in a wide working range. Moreover, from the obtained curve, the experimental stiffness of the sensing system (considering tip displacements) is derived and is equal to about $6.48 \mathrm{~N} / \mathrm{m}$. As such, linear 
approaches are used for the modeling. In this case, when a gripping force $F_{c}$ is applied at the tip of the sensing arm, the dynamic equation of the system is given as follow:

$F_{c}-\left(\frac{x_{e b}}{L}\right)^{2} \cdot \sigma_{b} \frac{\mathrm{d} y_{b}(L)}{\mathrm{d} t}-\left(\frac{x_{e b}}{L}\right)^{2} \cdot K_{l b} \cdot y_{b}(L)=\left(\frac{x_{e b}}{L}\right)^{2} \cdot M_{b} \frac{\mathrm{d}^{2} y_{b}(L)}{\mathrm{d} t^{2}}$

$\sigma_{b}$ and $M_{b}$ are respectively the damping factor, and the total mass of the sensing system, $K_{l b}$ is the linear stiffness of the suspensions and $x_{e b}=850 \mu m$ (see Fig6). The analytical expression of the stiffness is: $K_{l b}=\frac{48 \cdot E \cdot I_{s b}}{L_{s b}^{3}}$, with: $I_{s b}=\frac{l_{s b} \cdot e_{s b}^{3}}{12}$.

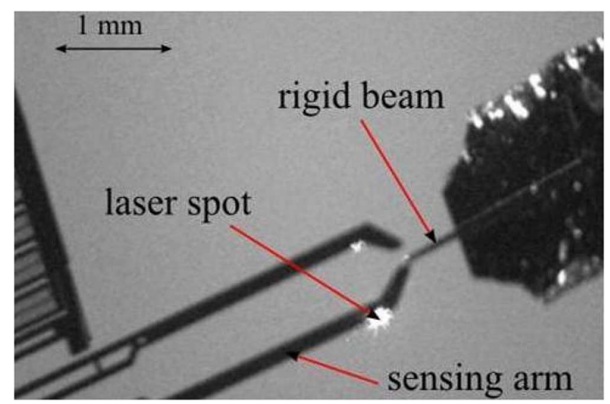

Fig. 7. Sensing arm excitation using a rigid beam

This leads to the state space equation:

$$
\begin{aligned}
& \left\{\begin{array}{l}
\left.\dot{X}_{b}=\left[\begin{array}{cc}
0 & 1 \\
-\frac{K_{l b}}{M_{b}} & -\frac{\sigma_{b}}{M_{b}}
\end{array}\right] \cdot X_{b}+\left[\begin{array}{l}
0 \\
y_{b}(L, t)=\left[\begin{array}{ll}
1 & 0
\end{array}\right] \cdot X_{b}
\end{array}\right)^{2} \cdot \frac{1}{M_{b}}\right] \cdot F_{c}
\end{array}\right. \\
& X_{b}=\left[\begin{array}{ll}
y_{b}(L, t) & \frac{d y_{b}(L, t)}{d t}
\end{array}\right]^{T}
\end{aligned}
$$

To extract the values of the damping and the mass of the sensing system, a parametric identification is performed using experimental data. To this end, we have generated a negative step force by pushing up the tip of the sensing arm with the previous rigid beam until a desired position and operating thereafter a fast withdrawal of the beam. The resulting motion (step response) of the arm tip (Fig9) is recorded using the laser interferometer. The real excitation force from the applied sensing arm thrust is deduced from the stiffness of the sensing system $(6.48 \mathrm{~N} / \mathrm{m})$. The sensing system has been excited with $12 \mu \mathrm{m}$ displacement from the punctual contact (i.e. $27 \mu m-15 \mu m$ ). Thus, $77 \mu N$ step force has been applied. Finally, using experimental data (Fig9) and a least squares identification method (Levenberg- Marquardt algorithm), unknown parameters have been identified such as: $M_{b}=0.66 \mathrm{mg}$, and $\sigma_{b}=0.71 \mathrm{mNs}^{2} / \mathrm{m}$.

Note that, the modeling of the sensing system is performed under the assumption that the electrostatic force generated by the capacitive sensor is negligible. This assumption is verified through the experimental data presented in Fig8. Indeed, the slope of the curve is close to the theoretical value of the spring constant $\left(\frac{x_{e b}}{L}\right)^{2} \cdot K_{l b}$ and hence the restoring force of the sensor is mainly due to the clamped-clamped beams.

\section{Coupled system modeling}

Actuation and sensing systems are coupled under the assumption that the grasped micro-object behaves as a spring

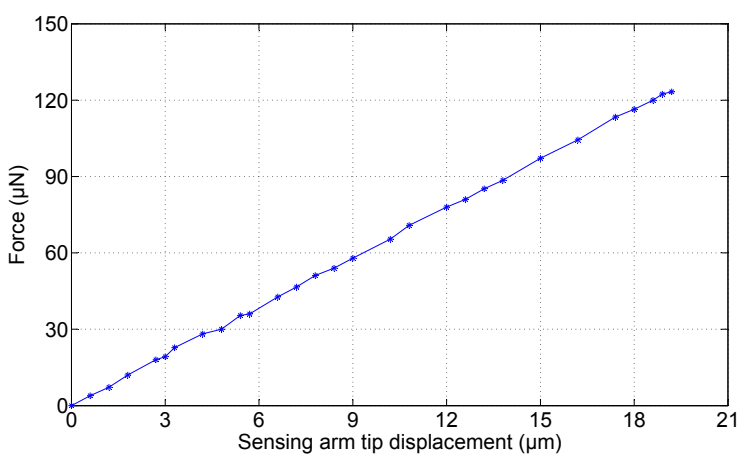

Fig. 8. Static gripping force/position characteristic of the sensing system

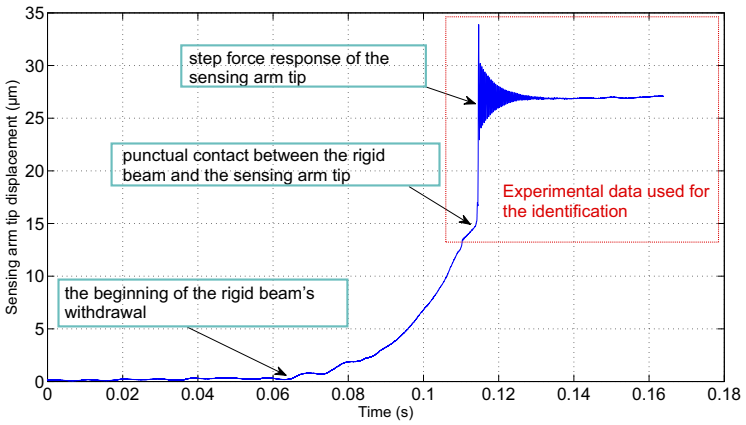

Fig. 9. Response of the sensing system at the tip of the sensing arm to a $77 \mu N$ step force. The displacement at the tip of the sensing arm (measured with the laser sensor) is in the opposite direction of $y$ axis. The zero value of the displacement is considered when starting the rigid beam's withdrawal.

with a stiffness $k_{0}$ as proposed in [22] [23]. The analytical expression of the gripping force is:

$$
F_{c}=k_{0}\left(\tilde{y}_{a}(L)-y_{b}(L)\right)
$$

The actuation system and the sensing system are then coupled using the analytical expression of the gripping force (15). The input of the coupled model is granted to the supply voltage $V_{i n}$ while the output relates to $F_{c}$. The state space equation of the coupled model is given as:

$$
\begin{aligned}
& \left\{\begin{array}{l}
{\left[\begin{array}{l}
\dot{X}_{a} \\
\dot{X}_{b}
\end{array}\right]=A_{\text {coup }} \cdot\left[\begin{array}{l}
\tilde{X}_{a} \\
X_{b}
\end{array}\right]+B_{\text {coup }} \cdot \tilde{V}_{\text {in }}} \\
F_{c}=C_{\text {coup }} \cdot\left[\begin{array}{l}
\tilde{X}_{a} \\
X_{b}
\end{array}\right]
\end{array}\right.
\end{aligned}
$$

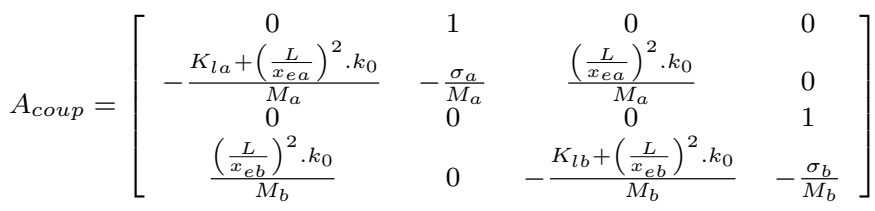

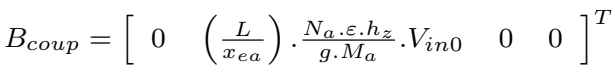

$$
\begin{aligned}
& C_{\text {coup }}=\left[\begin{array}{llll}
k_{0} & 0 & -k_{0} & 0
\end{array}\right]
\end{aligned}
$$

To identify the parameter $k_{0}$, a $10 \mathrm{~V}$ step voltage is applied to the gripper while handling the microball. Then, $k_{0}$ is identified using the least square identification method. Consequently, $11 \mu N$ gripping force is obtained in steady state and the algorithm found $k_{0}=174.5 \mathrm{~N} / \mathrm{m}$. Therefore, for $20 \mathrm{Khz}$ sampling 
frequency, the discrete canonical state space model of the coupled system is:

$$
\left\{\begin{array}{l}
X(k+1)=A \cdot X(k)+B \cdot V_{i n}(k) \\
F_{c}(k)=C \cdot X(k) .
\end{array}\right.
$$

$$
\begin{aligned}
& A=\left(\begin{array}{cccc}
0.8334 & 0.5196 & 0 & 0 \\
-0.5196 & 0.8334 & 0 & 0 \\
0 & 0 & 0.8348 & 0.5325 \\
0 & 0 & -0.5325 & 0.8348
\end{array}\right) \quad \mathrm{B}=\left(\begin{array}{c}
-0.1935 \\
0.3787 \\
0.1540 \\
0.5294
\end{array}\right) \\
& \mathrm{C}=\left(\begin{array}{cccc}
0.3779 & 0.4192 & 0.6764 & 0.0229
\end{array}\right) \times 10^{-6}
\end{aligned}
$$

Experiments shows that the noise at the output of the force sensor reaches $2.35 \mu N$. An optimal filter is required for increasing the force control performances when reaching the noise level. Indeed, low pass filtering allows improving the resolution of static and slowly varying forces but fast forces are filtered which is a real problem in our study. For this reason and in order to filter the measurement noise without loss of the system dynamics, we propose to use a Kalman filter.
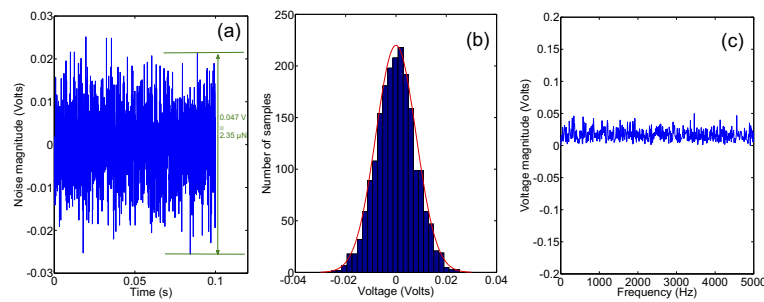

Fig. 10. Measurement noise: time evolution (a), distribution (b) and frequency spectrum $(c)$

\section{KALMAN FILTERING}

A discrete Kalman filter is designed and implemented in order to increase the signal to noise ratio of the measurement provided by the force sensor. The filter is tuned taking into account the covariance of measurement and process noises.

\section{A. Kalman filter}

The Kalman filter is based on a stochastic state space representation including a process noise $w(k)$ and a measurement noise $v(k)$. Noises are assumed to be independents, white, and with normal probability distributions. Considering the coupled system (17), the filter estimates the state $X \in \Re^{4 \times 1}$ using the noisy measurement $V_{\text {out }_{n o i s e}}$ and the input $V_{\text {in }}$ according to:

$$
\left\{\begin{array}{l}
X_{\text {noise }}(k+1)=A \cdot X_{\text {noise }}(k)+B \cdot V_{\text {in }}(k)+w(k) \\
V_{\text {out }_{\text {noise }}}(k)=S_{n}^{-1} . C \cdot X_{\text {noise }}(k)+v(k)
\end{array}\right.
$$

$X_{n o i s e}$ is the noisy state vector of the coupled system and $S_{n}=50 \mu N /$ volts is the sensitivity of the force sensor.

The Kalman filter recursively estimates the process state vector (and therefore the output) taking into account process and measurement noises in a way of minimizing the estimation error variance [24]. This is conducted following the repetition of two steps: time update and measurement update. In the time update, the a priori state (denoted $\hat{X}^{-}$) of the process is estimated according to the current state and error covariance. In the measurement update, the measurement is taken into account in order to obtain an improved state estimate leading to the a posteriori state (denoted $\hat{X}$ ). In practice, we assume that the covariance of the process noise and that of the measurement noise (required to compute the filter) remain constant during the manipulation task.

\section{B. Noise characterisation}

Noise characterization is done under the assumption that the process noise is mainly due to the input noise $v_{\text {input }}$ from the gripper supply in which a case $w(k)=B \cdot v_{\text {input }}(k)$ (see [25]). The measurement noise is recorded (using a dSPACE control board) from the output of the force sensor when no mechanical input is applied on the tip of the sensing arm. Experiments are done under different environmental conditions. Here we only present the obtained results for ambient conditions (i.e. 23 degrees temperature and 1.013 bar pressure).

As a result of measurement noise analysis, a Gaussian distribution with a mean of zero is observed (Fig10b) and the covariance is estimated at $6.33 \times 10^{-5} V^{2}$. Moreover, this noise is considered as white due to its frequency spectrum (Fig10c). Also, the input noise is measured at the output of the gripper supply when 0 Volts is applied. It has been shown that the input noise is also white, centered and has a normal distribution with a covariance of $9.62 \times 10^{-5} \mathrm{~V}^{2}$.

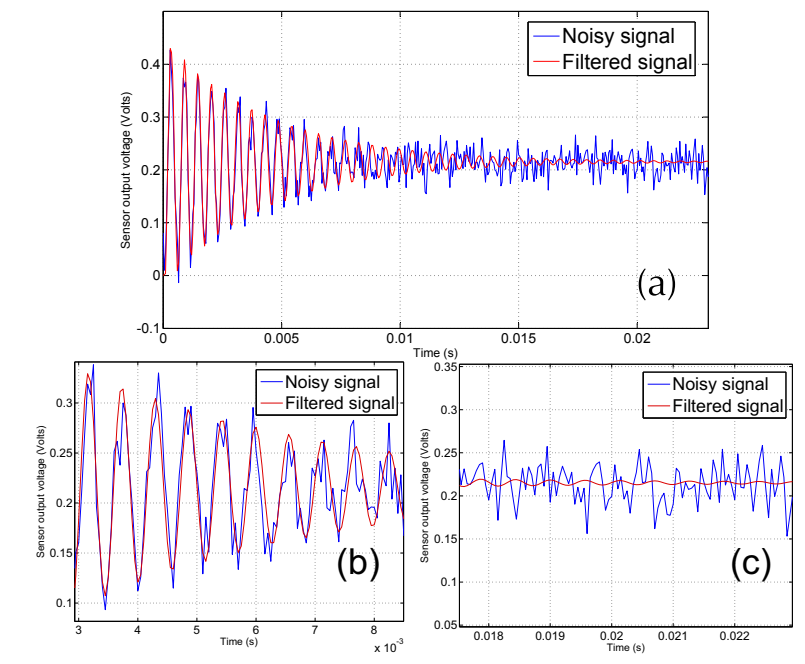

Fig. 11. Comparison between the noisy measurement and the filtered one (a): dynamic (b) and static (c) modes

Process and measurement noises are considered in our study as independent from each other because they are generated by different devices. Therefore, from the obtained measurement and input noises convariances, the Kalman gain $K_{e}$ has been calculated offline and its steady state has been implemented in real time for filtering. We have then implemented the folowing gain: $K_{e}=\left[\begin{array}{llll}-0.11 & 0.06 & 0 & -0.008\end{array}\right]^{T}$

\section{Results}

The Kalman filter has been implemented in real time with a $20 \mathrm{KHz}$ sampling frequency using the Matlab/Simulink software $(r 2007 b)$ and a dSPACE control board, while handling the micro-glass ball. Noisy and filtered measurements in response to a $10 \mathrm{~V}$ step voltage (around $60 \mathrm{~V}$ ) are presented in (Fig11a). 
A significant decrease of the measurement noise is observed (Fig11c) without loss of dynamic behaviour measurement (Fig11b). The Kalman filter allows a reduction of about $97 \%$ of the measurement noise. In order to verify the effectiveness of obtained results, a comparison between the real gripping force and the one estimated by the Kalman filter is done later. The laser interferometer is used to measure the deflection at the tip of the sensing arm while the gripper is applying a gripping force. This experiment allows extracting the real griping force thanks to a good knowledge of the sensing system's stiffness. The estimation of the real griping forces using the gripper's sensor and the Kalman filter will lead to get accurate micromanipulation task through the force controller.

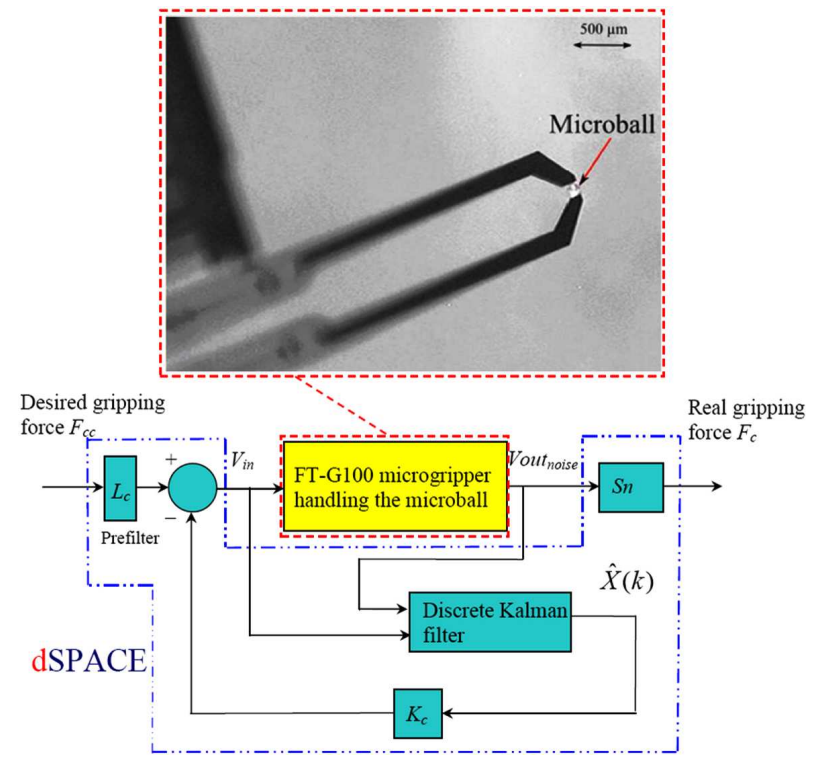

Fig. 12. Block diagram of force-controlled microgripping

\section{FEedBACK CONTROL}

For the manipulation of the glass ball, the gripper is manually positioned so that the tip of the sensing arm is in contact with the ball. Thereafter, the actuated arm is supplied with a $60 \mathrm{~V}$ voltage to come into contact with the ball. We choose to apply a controlled griping force of $10 \mu N$. The applied force must be very fast with no overshoot during transient periods (fast response time) allowing an efficient gripping of the manipulated object when performing for instance high speed pick and place tasks. In our study, the microgripper is not moving, however for further applications we desire a force response time lower than $10 \mathrm{~ms}$. Therefore, we propose to use a Linear Quadratic Gaussian (LQG) control algorithm because it is well suited when trade-off between closed loop performances and output noise reduction has to be taken into account. The LQG control synthesis includes a Kalman filter and allows the computation of the filter and the controller independently thanks to the separation principle.

The LQG problem attempts to find a controller that provides the best performances with respect to a given optimal energy criterion. Fig 12 shows the block diagram of the force control system with a feedback vector $K_{c}$ to correct the dynamic performances and a prefilter $L_{c}$ for the static performances. Considering the following energy criterion:

$$
J(k)=\sum_{i=k}^{\infty} X^{T}(i) \cdot Q \cdot X(i)+V_{i n}^{T}(i) \cdot R \cdot V_{i n}(i) .
$$

$Q$ and $R$ are the weighting given to the state and the control voltage in the optimal criteria. The dynamics of the closed loop system can be adjusted by an appropriate choice of the weighting parameters. Therefore, the linear quadratic problem focuses on searching the optimal feedback vector $K_{c}$ which minimizes the energy criteria with respect of the Lyapunov stability. For a discrete case, this vector is given by [26]:

$$
K_{c}=\left(R+B^{T} \cdot P_{c} \cdot B\right)^{-1} \cdot B^{T} \cdot P_{C} \cdot A
$$

$P_{c}$ is the solution of Riccati equation. In the steady state, we obtain:

$$
F_{c}(k)=C \cdot\left[\text { eye }(n)-\left(A-B \cdot K_{C}\right)\right]^{-1} \cdot B \cdot L_{c} \cdot F_{c c}(k)
$$

Moreover, in order to get the desired gripping force in the static mode (i.e. $F_{c c}(k)=F_{c}(k)$ ), the prefilter is chosen as:

$$
L_{c}=\left[C \cdot\left[\text { eye }(n)-\left(A-B \cdot K_{C}\right)\right]^{-1} \cdot B\right]^{-1} .
$$

In dynamic mode, weighting parameters $R$ and $Q$ have been selected to reach wanted closed loop performances using the Bryson method [27]. This method allows defining upper limitations of the gripping force and the supply voltage. Thus:

$$
\begin{aligned}
& R=\frac{1}{\sup \left(\tilde{V}_{i n}\right)^{2}}, \quad Q=\operatorname{diag}\left[\frac{1}{\sup \left(x_{1}\right)^{2}}, \frac{1}{\sup \left(x_{2}\right)^{2}}, \frac{1}{\sup \left(x_{3}\right)^{2}}, \frac{1}{\sup \left(x_{4}\right)^{2}}\right] \\
& {\left[\begin{array}{llll}
\sup \left(x_{1}\right) & \sup \left(x_{2}\right) & \sup \left(x_{3}\right) & \sup \left(x_{4}\right)
\end{array}\right]^{T}=\sup (X)}
\end{aligned}
$$

The performances needed in our study are given as follow: $\sup \left(V_{i n}\right)=10 \mathrm{~V}$ and $\sup \left(F_{c}\right)=10 \mu N$ (no-overshoot).

Let us recall that the discrete state space model (17) of the coupled system is in the modal canonical form. In such a representation, the real eigenvalues of the actuation system and those of the sensing system appear separately on the diagonal of the state matrix. As such, the sup functions $\sup \left(x_{1}\right)$ and $\sup \left(x_{2}\right)$ can be selected independently of $\sup \left(x_{3}\right)$ and $\sup \left(x_{4}\right)$. Thus, according to (17), we have: $\sup \left(F_{c}\right)=C \cdot \sup (X)$. The latter equation has no unique solution. Many combinations of $\sup (X)$ allow obtaining the desired $\sup \left(F_{c}\right)$. Therefore, in order to simplify the determination of the sup functions $\sup \left(x_{i}\right)$, we have initially chosen $\sup \left(x_{1}\right)$ equal to $\sup \left(x_{3}\right)$ and $\sup \left(x_{2}\right)=\sup \left(x_{4}\right)=0$. In other word, we apply the same weigh to only one state of the actuation system and one state of the sensing system. In this case, we obtain: $\sup \left(x_{1}\right)=$ $\sup \left(x_{3}\right)=9.5$. Then, the values of $\sup \left(x_{2}\right)$ and $\sup \left(x_{4}\right)$ are refined by simulation (feedback control) and we have selected: $\sup \left(x_{2}\right)^{2}=0.1 \times \sup \left(x_{1}\right)^{2}$ and $\sup \left(x_{4}\right)^{2}=0.001 \times \sup \left(x_{3}\right)^{2}$. Finally: $\sup (X)=\left[\begin{array}{llll}9.5 & 3 & 9.5 & 0.3\end{array}\right]^{T}$. So, the weighting parameters are given as: $R=1 \quad Q=\operatorname{diag}[1,10,1,1000]$. Which leads to: $K c=\left[\begin{array}{llll}-0.0132 & -0.0001 & -0.8921 & 1.5947\end{array}\right]$

\section{SYSTEM SETUP}

The micro-manipulation station (Fig13) is made up of a 3-DOF manual micro-positioning table (M-UMR 5.16, Newport) where the FT-G100 microgripper is fixed and tilted with an angle of $45^{\circ}$ to allow gripping the samples. Due to the small size of 
the gripper, a microscope is used and all the components are mounted on a vibration isolation table. The control algorithm is developed using the Matlab/Simulink software (r2007b) and is implemented in real time using a dSPACE control board with a sampling frequency of $20 \mathrm{Khz}$.

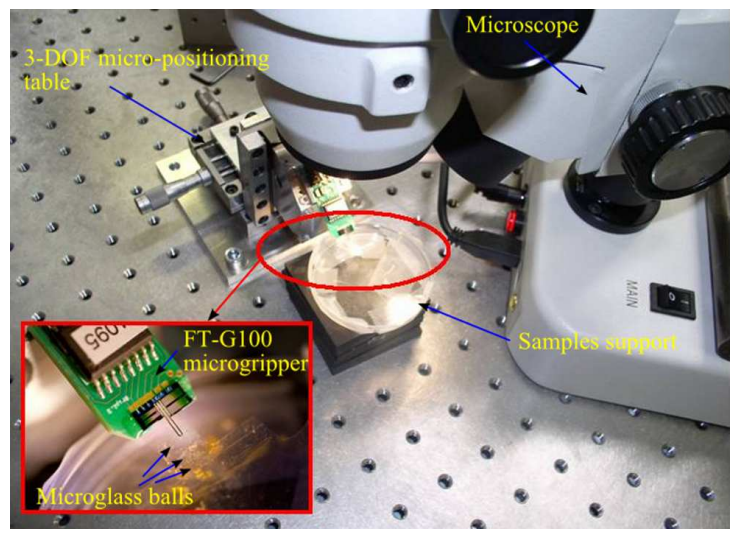

Fig. 13. Force-controlled micromanipulation setup

\section{EXPERIMENTAL RESULTS AND DISCUSSION}

The $80 \mu \mathrm{m}$ glass ball has been handled successfully with $10 \mu N$ gripping force. Fig 14 and Fig 15 show that the desired control performances are obtained, i.e. the response time of the gripping force is reaching $6 \mathrm{~ms}$ and no overshoot is detected. The voltage doesn't exceed $10 \mathrm{~V}$ around the operating point. A small peak is also observed on the actuation voltage dynamic at approximately $1.5 \mathrm{~ms}$ (Fig15) giving the impulse to the gripping force and inducing a fast response time. Moreover, we can observe the high quality of the griping force estimated by the Kalman filter which has extracted a precise signal from the noisy output of the sensor.

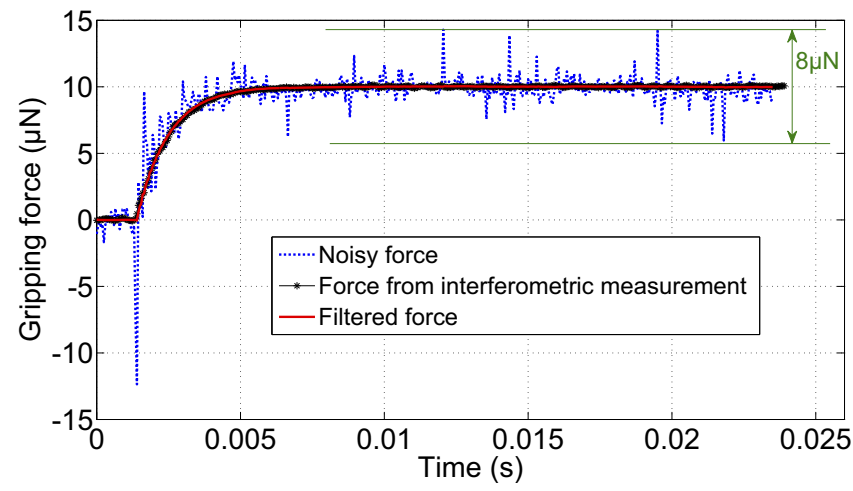

Fig. 14. Force-controlled microgripping for $10 \mu N$ force reference

Nevertheless, it is important to know whether the filtered signal reflects the real gripping force. Thus we have recorded the micro-motions on the tip of the sensing arm while applying the force control in order to deduce the real gripping force dynamic through the knowledge of the sensing system stiffness. Fig 14 shows both the force estimated by the Kalman filter and the deduced one, the error between the two signals is less than $0.1 \mu N$. The effectiveness of the Kalman filter is then entirely proven.

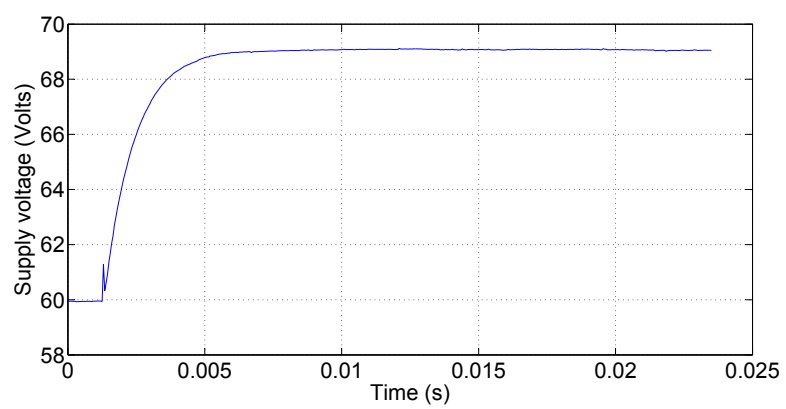

Fig. 15. Evolution of the control voltage for $10 \mu N$ force reference

\section{CONCLUSION}

In this paper, we have presented a knowledge based modeling and a gripping force control of an electrostatic microgripper with integrated force sensor. A nonlinear model of the actuation system has been proposed and validated experimentally in a large working range and limit of linear behavior has been extracted. For gripping force control, a state space model has been developed coupling the dynamics of the actuation and the sensing systems through the manipulated object stiffness which has been identified using experimental measurements. In this case, a linear model of the actuation system has been extracted from the nonlinear modeling around $60 \mathrm{~V}$ actuation voltage. The control algorithm based on a LQG algorithm has been implemented and tested for handling $80 \mu \mathrm{m}$ glass balls. The Kalman filtering allowed increasing the gripping force resolution that was limited by the noise level. Indeed, a reduction of $97 \%$ of the measurement noise has been obtained. The effectiveness of the estimated force has been proved by comparison with results from an external interferometer. Our future work will concern the control of the actuation system in a wide working range taking into account dynamic changes due to the non linear behavior.

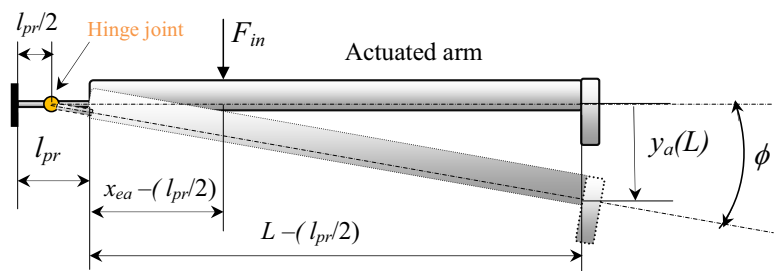

Fig. 16. Pseudo rigid body consisting of the actuated arm and the flexure joint.

\section{APPENDIX A}

\section{CALCULATION OF THE LATERAL STIFFNESS OF A PSEUDO} RIGID BODY

Consider the actuated arm of the microgripper subject to an external force $F_{i n}$ applied at a distance $\left(L-x_{e a}\right)$ from the free end (Fig16). The pseudo rigid body consists of two beam segments; the first one must be significantly shorter and more flexible than the second segment. This implies that $l_{p r} \ll L$ and E.I $I_{p r} \ll E . I$. Where: $I=1.4 \times 10^{-17} \mathrm{~m}^{4}$ and $I_{p r}=7.2 \times 10^{-21} \mathrm{~m}^{4}$ are the area moment of inertia of the short and the large beam segments respectively. Moreover $l_{p r}=300 \mu m$ (see Fig1). 
The torque $T$ required to deflect the free end of the actuated arm with an angle $\phi$ is:

$$
T=\frac{E \cdot I_{p r}}{l_{p r}} \cdot \phi
$$

With:

$$
T=x_{e a} \cdot F_{i n}
$$

Moreover, for a small angle of deflection $\phi$

$$
y_{a}(L)=L \cdot \phi
$$

The lateral stiffness of the pseudo rigid body is then given as:

$$
K_{p r}=\frac{F_{i n}}{y_{a}(L)}=\frac{E \cdot I_{p r}}{l_{p r} \cdot x_{e a} \cdot L}
$$

This model is more accurate when the bending is the dominant loading in the flexure joint. For more information, refer to [28] (page 139).

\section{REFERENCES}

[1] Clevy C, Hubert A, Chaillet N, "Flexible micro-assembly system equiped with an automated tool changer", Journal of Micro-Nano Mechatronics, vol.4, no.1-2, pp.59-72, 2008.

[2] Sun Y, Wan K T, Roberts K P, Bischof J. C, Nelson B J, "Mechanical property characterization of mouse zona pellucida",IEEE Trans Nanobioscience,vol.2, pp.279-286, 2003.

[3] Liu X Y, Kim K Y, Zhang Y, Sun Y, "Nanonewton force sensing and control in microrobotic cell manipulation", International Journal of Robotics Research, vol.28, no.8, pp.1065-1076, 2009.

[4] R Perez, Agnus J, Clevy C, Hubert A, Chaillet N, "Modelling, fabrication and validation of a high performance 2 DOF piezoactuator for manipulation.”,IEEE/ASME Trans on Mechatronics , vol.10, issue.2, pp.161-171, 2005.

[5] Lan C. C, Lin C. M, Fan, C. H, "A Self-Sensing Microgripper Module With Wide Handling Ranges", IEEE/ASME Trans on Mechatronics, vol.16, issue.1, pp.141-150, 2011

[6] Kim D. H, Lee M. G, Kim B, Sun Y, "A superelastic alloy microgripper with embedded electromagnetic actuators and piezoelectric force sensors: a numerical and experimental study ", Smart Materials and Structures, vol.14, no.6, pp.12651272, 2005.

[7] Kemper M, "Development of a tactile low-cost microgripper with integrated force sensor", Proc. IEEE ICCA, 2004, Taipei, Taiwan.

[8] Agarwal N, Aluru N R, "A stochastic Lagrangian approach for geometrical uncertainties in electrostatics", J. Comput. Phys, Vol.226, issue.1, pp.156-179, 2007.

[9] Park J, Kim S, Kim D H, Kim B, Kwon S, Park J O, Lee K I, ”Advanced Controller Design and Implementation of a Sensorized Microgripper for Micromanipulation", Proc. IEEE ICRA, 2004, New Orleans, USA.

[10] Carrozza M C, Eisinberg A, Menciassi A, Campolo D, Micera S, Dario $\mathrm{P}$, "Towards a force-controlled microgripper for assembling biomedical microdevices", J. Micromech. Microeng, vol.10, pp.271276, 2000.

[11] Kyung J H, Ko B G, Ha Y H, Chung G J 'Design of a microgripper for micromanipulation of microcomponents using SMA wires and flexible hinges ", Sens and Act A: Physical, vol.141, issue.1, pp.144-150, 2008.

[12] Kim K, Liu X, Zhang Y, Sun Y, "Micronewton force-controlled manipulation of biomaterials using a monolithic MEMS microgripper with two-axis force feedbacky", Proc. IEEE ICRA, Pasadena, CA, USA,2008.

[13] Kim K, Xinyu L, Yong Z, Sun Y, ”Nanonewton force-controlled manipulation of biological cells using a monolithic MEMS microgripper with two-axis force feedback", J. Micromech. Microeng, vol.8, no.5, 2008.

[14] Boudaoud M, Haddab Y, Le Gorrec Y. , "Modelling of a MEMS-based microgripper: application to dexterous micromanipulation", Proc. IEEE IROS 2010, Taipei, Taiwan.

[15] MS3110 universal capacitive readoutTM IC, Irvine Sensors Corp, 2004.

[16] Beyeler F, Neild A, Oberti S, Bell D, Yu S, Dual J, Nelson B, "Monolithically fabricated microgripper with integrated force sensor for manipulating microobjects and biological cells aligned in an ultrasonic field" Journal of microelectromechanical systems, vol.16, pp.7-15, 2007.

[17] Moussa W. A, Ahmed H, Badawy W, Moussa M, "Investigating the reliability of electrostatic comb-drive actuators used in microfluidic and space systems using finite element analysis", in Can. J. Elect. Comput. eng, vol.27, no.4, pp.195-200, 2002.
[18] Legtenberg R, Groeneveld A, Elwenspoek M, "Combdrive actuators for large displacements", J. Micromech. Microeng, vol.6, pp.320-329, 1996.

[19] Frisch-Fay R 1962 Flexible bars (London: Butterworths).

[20] Gere J M and Timoshenko S P 1987 Mechanics of Materials 2nd edn (Van Nostrand Reinhold)

[21] Borg S. F, "Fundamentals of Engineering: Elasticity". World Scientific Pub Co Inc, 1990. ISBN-10: 9810201656.

[22] Eppinger S D, Seering W P, "On dynamic models of robot force control.", Proc. IEEE ICRA, 1986.

[23] Rakotondrabe M, Haddab Y, Lutz P, "Modelling and control of a highly modular microassembly system". in IWMF, Shanghai, China, 2004.

[24] Kalman R. E, "A new approach to linear filtering and prediction problems", Trans ASME. Journal of Basic Engineering, pp.35-45, 1960.

[25] Haddab Y, Chen Q, Lutz, P, "Improvement of strain gauges micro-forces measurement using Kalman optimal filtering", Mechatronics, vol.19, issue.4, pp.457-462, 2009.

[26] Anderson B. D. O, Moore, J. B, "Optimal control: linear quadratic methods", Prentice Hall, 1990

[27] Bryson A. E, Ho Y. C, "Applied optimal control”, Hemisphere, 1975

[28] Howell L. L, "Compliant mechanisms". John Wiley Sons Limited, 2001.

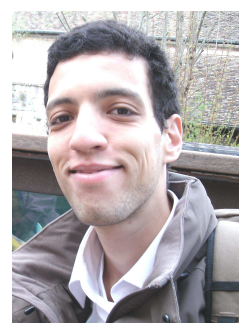

Mokrane Boudaoud received the Engineering degree in Automatic Control from the University of Science and Technology Houari Boumédiène (USTHB, Algiers, Algeria), and a MS degree in Mechatronics and Microrobotics at the University of Franche-Comté (UFC, Besançon, France) in 2009. $\mathrm{He}$ is currently a PhD student at AS2M (Automatic Control and Micro-Mechatronic Systems) department of Femto-st Institute. His current research interests include modeling and robust control of electrostatically actuated MEMS (Micro Electro Mechanical Systems) based microgrippers, Atomic Force Microscope (AFM) cantilevers calibration and noise characterization in micromanipulation systems.

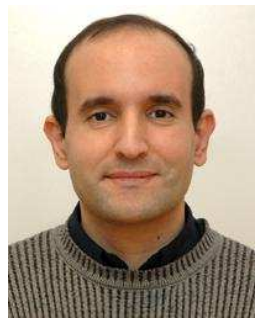

Yassine Haddab received the Engineering degree in electrical engineering from the university of TiziOuzou, Algeria, the M.S. degree from ENSMM, Besanon, France and the Ph.D. degree from the University of Franche-Comté, Besanọn, France, in 2000. His work dealt with the design, the modeling and the control of micromanipulation systems. Since 2002, he is an Associate Professor with ENSMM, Besanon where he teaches control theory, real time control and microrobotics. His research interests include the design and control of high precision microrobots and microsystems. He also contributes to the development of new microrobots architectures.

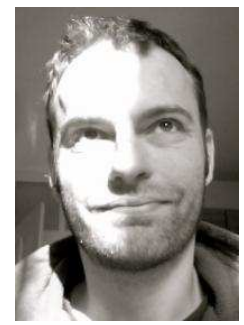

Yann Le Gorrec was graduated as engineer in "Control, Electronics, Computer Engineering" at the National Institute of Applied Sciences (INSA Toulouse, France) in 1995. He received in 1998 his $\mathrm{Ph}$. D. degree from the National Higher School of Aeronautics and Aerospace (Supaero, Toulouse, France). His field of interest was robust control and self scheduled controller synthesis. From 1999 to 2008, he was Associate Professor in Automatic Control at the Laboratory of Control and Chemical Engineering of Lyon Claude Bernard University (LAGEP, Villeurbanne, France). He worked on modelling of physico-chemical processes, robust control, modelling and control of distributed parameter systems. From september 2008 he is Professor at National Engineering Institute in Mechanics and Microtechnologies. His current field of research is control of smart material based actuators, distributed micro systems and more generaly control of micro actuators. 